\title{
Treatment outcomes for elderly patients in Thailand with pulmonary tuberculosis
}

\author{
Wilawan Somsong ${ }^{1,2}$, Saranath Lawpoolsri' ${ }^{1}$, Yuthichai Kasetjaroen², Weerawat Manosuthi ${ }^{3}$ \\ Jaranit Kaewkungwal1,**
}

\begin{abstract}
Background: Elderly patients with pulmonary tuberculosis are less likely to achieve treatment success than younger patients, and patients aged $\geq 60$ years have a substantial increase in mortality.

Objectives: To compare treatment outcomes over 2 periods during the transition of Thai national tuberculosis (TB) reporting systems and determine treatment success rates and mortality for elderly patients in TB treatment-care settings in Thailand.

Methods: Retrospective cohort study of all records of elderly patients extracted from 2 national TB databases in Thailand: the TB Case Management (TBCM) database of the National TB Program (2014-2015) and the database of the National Health Security Office (NHSO; 2010-2011).

Results: There were 8,301 elderly patients with TB in the TBCM cohort and 11,869 in the NHSO cohort. Overall treatment success rates were $78.5 \%$ for patients in the TBCM cohort and $87.5 \%$ for patients in the NHSO cohort. High success rates for treatment were found for those aged 60-69 years: 91.1\% in 2010-2011 and 85.0\% in 2014-2015. High mortality was reported for patients aged $\geq 90$ years: $34.6 \%$ in $2010-2011$ and $50.0 \%$ in $2014-2015$.

Conclusions: Compared with the NHSO historical cohort, success rates for treatment were lower and death rates were higher in the TBCM cohort. Because NHSO enforced intensive case monitoring and follow-up while TBCM has no such mechanism, the estimates from the TBCM database may be less accurate for TB circumstances in Thailand. Frequent routine home visits may ensure more complete treatment-care information and support, and increase the treatment success rate in the elderly.
\end{abstract}

Keywords: aged, drug therapy, epidemiology, pulmonary, Thailand, tuberculosis

Pulmonary tuberculosis (PTB) is emerging as a significant health problem among the elderly in Thailand. The incidence of PTB in Thailand was estimated at 137 per 100,000 population in 2010 and 172 per 100,000 population in 2015 [1, 2]. According to a national report, the notification rate for a new positive acid-fast bacilli sputum smears in the group patients aged $\geq 65$ years appeared to be the highest compared with that of other age groups, increasing from 132.5 per 100,000 population in 2008 to 134.6 per 100,000 population in 2009 [3]. In 2016, about $23.2 \%$ of all cases of PTB registered were in patients aged $\geq 65$ years [4].

PTB in the elderly is more likely to present with dyspnea, nonspecific symptoms, and an atypical radiographic appearance [5]. Problems for the elderly with TB include delays in

*Correspondence to: Jaranit Kaewkungwal, Department of Tropical Hygiene, Faculty of Tropical Medicine, Mahidol University, Bangkok 10400, Thailand, e-mail: jaranit.kae@mahidol.ac.th

'Department of Tropical Hygiene, Faculty of Tropical Medicine, Mahidol University, Bangkok 10400, Thailand

${ }^{2}$ Bureau of Tuberculosis, Ministry of Public Health, Bangkok 10120, Thailand

${ }^{3}$ Department of Medicine, Bamrasnaradura Infectious Disease Institute, Ministry of Public Health, Nonthaburi 11000, Thailand

¿ Open Access. ๑ 2018 Wilawan Somsong et al., published by Sciendo. (co) BY-Nc-ND This work is licensed under the Creative

Commons Attribution NonCommercial-NoDerivatives 4.0 License. 
diagnosis and treatment-related issues. Key problems have included poor compliance with treatment, poor tolerance of drug therapy, and the presence of underlying or associated diseases [6,7]. The main cause of failure of treatment for PTB, at whatever age, is related to poor patient compliance, and this problem is particularly accentuated in the elderly. A study in Taiwan found that patients aged $\geq 60$ years with extensive radiographic disease presentation were associated with unfavorable outcomes [8]. A study in Hong Kong found that factors independently associated with unfavorable outcomes of patients with PTB included age $\geq 65$ years with moderate-to-extensive disease and positive acid-fast bacilli sputum smears [9]. A study in Korea found that cases of PTB in elderly patients showed higher frequencies of adverse drug reactions: $40.7 \%$ in 2005 and $32 \%$ in 2009 [10]. Similarly, a study in China suggested that the main reason for interrupted treatment mentioned by patients was the presence of adverse effects during treatment (46.5\%); and such interruption of treatment was found to be highly associated with noncure outcome $[11,12]$.

In Thailand, the treatment success rates for patients with TB for all age groups were $86.0 \%$ (2010), 85.0\% (2011) [13], $82.3 \%$ (2014), and $80.9 \%$ (2015) [14]. Mortality rates for all age groups of patients with TB were 7.0\% (2010), 7.0\% (2011) [13], 7.7\% (2014), and 8.0\% (2015) [14]. According to a report in 2015, PTB treatment success rates and mortality of patients aged $\geq 60$ years compared with those aged $<60$ years differed; the treatment success rates were $74.3 \%$ vs. $79.1 \%$ and mortality were $14.7 \%$ vs. $5.5 \%$, respectively [4]. These problems have raised concern by the Thai National TB Program because of the high notification rate for elderly patients with PTB and the apparent inability to increase treatment success rate for PTB cases, particularly in the elderly. The routine TB report from the Thai National TB program categorizes elderly patients as those aged $>60$ or 65 years. There has been no detailed empirical evidence about treatment outcomes, focusing on success and mortality among elderly.

Therefore, the objective of the present study was to investigate treatment success and mortality rates in various age and sex subgroups of TB patients. Because of the changes in case management and reporting mechanisms to the Thai National TB program, this study collected data from 2 national databases in Thailand. The Thai TB Case Management (TBCM) database contains data that are collected by TB clinic units; the clinics are required to compile information from individual patients and enter it into the database program and submit to 3 upper levels: the provincial health offices, the Office of Disease Control and Prevention, and the Thai National TB Program. The other data source was the Thai National Health
Security Office (NHSO) database that contains data collected at the hospital level, which is required to compile information from individual patients for cost reimbursement. The other primary objective of this study was to compare the differences in identification of TB treatment outcomes among elderly in the 2 databases over the 2 periods, TBCM (2014-2015) and NHSO (2010-2011).

\section{Methods}

\section{Study design}

We conducted a retrospective cohort observational study, which we report here according to the Strengthening the Reporting of Observational Studies in Epidemiology (STROBE) Statement (http://www. strobe-statement.org/).

\section{Ethics approval}

The deidentified data were collected from the TB databases of the TBCM and NHSO. Access to the records was permitted by an authorized person for each organization. The study was reviewed and approved by the ethics committee of the Faculty of Tropical Medicine, Mahidol University (certificate No. MUTM 2013-026-01-04).

\section{Study setting and data sources}

This study utilized data from the 2 national TB electronic case-based systems in Thailand, the TBCM and NHSO. We extracted records from the 2 databases representing 2 cohorts; the TBCM cohort comprised TB patients during 2014-2015. Data in the TBCM are maintained by the Thai National TB Program or Bureau of Tuberculosis (BTB), which is the organization responsible for disease control programs. The data from the BTB are routinely analyzed and sent as feedback at regional, provincial, and hospital levels. As a historical comparison group, the NHSO cohort included data from patients with TB during 2010-2011. The NHSO is the organization responsible for health insurance. Under the reimbursement mechanism, hospitals are required to complete and submit TB reports to the NHSO. In an attempt to create a single national TB database system in Thailand, there has been a transition period for the TBCM and reporting systems at a national level, and recently, the TBCM and NHSO databases were reconciled. 


\section{Participants}

The present study focused on all new TB patients aged $\geq 60$ years with positive acid-fast bacilli sputum smears, who were registered for first-line drugs for PTB treatment with a 6- to 9-month course of chemotherapy. The study focused on TB patients with new positive smears because they can spread TB infection to other person, and thus, it is important to increase treatment success among this group. The follow-up period for each patient was 12 months. Data from the TBCM from October 1, 2013, to September 30, 2015 (fiscal year 2014-2015), were extracted. During this period, the data came from 32 hospitals in 2014 and 836 hospitals in 2015, covering 9,451 patients. The data from the NHSO from October 1, 2009, to September 30, 2011 (fiscal year 2010-2011), were extracted from records from all 859 hospitals registered under the Bureau of Policy and Strategy of the Thai Ministry of Public Health, covering 16,335 patients.

\section{Variables}

The independent variables were the year of TB treatment registration (data sources), and age and sex subgroups of patients with TB. The dependent variable was the treatment outcome focusing on treatment success and mortality.

\section{Data collection and management}

Data obtained from the TBCM and NHSO were retrieved by individuals authorized to access the 2 systems. The personal information of each patient was deidentified, but outcomes were still monitored throughout the follow-up visits during the treatment period with the newly assigned study identification number. In both databases, treatment outcomes were extracted from the databases for each patient with PTB who started their treatment in the previous 12 months. A followup of treatment outcome at 12 months was conducted to allow sufficient time to obtain complete data recorded in the databases regarding treatment outcome. Once a person completed treatment, outcome was categorized in 1 of 5 categories (success, failure, died, loss to follow-up, and transferred out) [15].

The treatment outcomes were classified according to 2009 WHO guidelines [16]. Successful treatment was defined as the patients with cases of PTB who successfully completed treatment, whether using bacteriological evidence of success ("cured") or without ("treatment completed") [17]. Failure was defined as a new patient whose culture or sputum smear microscopy was positive for acid-fast bacilli at the end of the 5th month or later during treatment. Death was defined as a patient who died from any cause during the course of treatment. Lost to follow-up was defined as a patient who did not start treatment or whose treatment was interrupted for 2 consecutive months or more [15]. Transferred out was defined as a patient who was transferred to a health facility in another basic management unit and for whom treatment outcome was unknown.

For the purpose of the present study, treatment outcomes were focused on treatment success and death. Successful treatment was defined as the sum of cured and completed treatment [18]. Unsuccessful treatment was defined as unfavorable outcome or the sum of treatment failure, deaths, loss to followup, and transfer out to other services [18]. Death from any cause during the course of treatment was extracted as originally coded in the databases.

\section{Statistical analyses}

Treatment outcomes (success and mortality) for elderly patients with PTB were analyzed by different age subgroups, sex, and year of treatment registration. The patient age was divided into 4 subgroups with the following ranges: $60-69,70-79$, $80-89$, and $\geq 90$ years. Treatment outcomes of the 4 age subgroups, sex, and year of treatment registration are presented descriptively and compared using a binomial regression model [19]. As this study was based on large cohorts following up elderly patients with PTB from treatment initiation to treatment outcome, the adjusted risk ratios (aRRs) and $95 \%$ confidence interval (CI) estimated by the binomial regression were calculated for the treatment outcomes of interest using all variables in the equation.

\section{Bias}

As the present study was conducted using secondary data from the 2 Thai national data sources, the typical data quality, particularly the completeness of the data, was the major source of bias. Nevertheless, data from all registered cases with complete and incomplete treatment outcomes were extracted from the 2 databases by authorized personnel and thus represented the evidence-based data at the national reporting systems. There were different monitoring and evaluation processes of the 2 national offices (TBCM-BTB and NHSO), which might, in turn, affect the data completeness and quality. 


\section{Results}

\section{Completeness of records of elderly patients with PTB in the databases}

Data within the TBCM during fiscal year 2014-2015 were obtained from 9,451 elderly patients with PTB. Among them, 1,150 were excluded due to change in diagnosis, no sputum result, or because no treatment outcomes were recorded. The final complete records were for 8,301 patients are as shown in Figure 1. From the NHSO database during fiscal year 2010 2011, for 16,335 elderly patients with PTB, 16,321 records had been correctly recorded for patients with age $\geq 60$ years. Of these patients, 15,833 had a new positive acid-fast bacilli sputum smear and 11,869 had PTB treatment outcomes available for review as shown in Figure 1.

\section{Treatment outcomes among different age groups of elderly patients with PTB}

The records in the TBCM revealed that among 8,301 elderly patients with $\mathrm{PTB}$, the overall cases with treatment success was $6,519(78.5 \%)$, those with treatment failure was $81(1.0 \%)$, those with death was $1,325(16.0 \%)$, those with loss to followup was $153(1.8 \%)$, and those with transferred out was 223 $(2.7 \%)$. The treatment success rate was $85.0 \%$ for those aged 60-69 years, and the mortality was $50.0 \%$ for elderly aged $\geq 90$ years. Analysis of the historical data in the NHSO databases revealed that among a total of 11,869 elderly patients with PTB, the treatment success was $10,388(87.5 \%)$, treatment failure was $183(1.5 \%)$, death was $976(8.2 \%)$, loss to followup was $163(1.4 \%)$, and transferred out was 159 (1.3\%). The treatment success rates for elderly patients with PTB in the historical database was $91.1 \%$ for those aged 60-69 years, and the mortality was $34.6 \%$ for those aged $\geq 90$ years as shown in Figure 2 .

\section{Comparisons of treatment outcomes among different groups of elderly with PTB}

Treatment outcomes for elderly patients with PTB in the 2 databases were significantly different. The treatment success rate in the 2014-2015 cohort (TBCM) was lower than that in the 2010-2011 historical comparison cohort (NHSO), 78.5\% vs. $87.5 \%$, respectively (Table 1). The treatment success rate for patients in the TBCM cohort was about $9 \%$ percentage points lower than that for those in the NHSO cohort $(\mathrm{aRR}=0.91,95 \%$ CI $0.90-0.92)$. Mortality in the 2014-2015 cohort (TBCM) was higher than that in the 2010-2011 historical comparison cohort (NHSO), $16.0 \%$ vs. $8.2 \%$, respectively (Table 2). The mortality of patients in the TBCM cohort was about 2 times than that in the NHSO cohort $(\mathrm{aRR}=2.01,95 \%$ CI 1.86-2.17).

Significant differences were found between treatment success rates for PTB patients in the 4 age subgroups $(P<$ 0.001). Compared with patients aged 60-69 years, those aged 70-79 years had a lower aRR or chance of treatment success at 0.94 (95\% CI $0.93-0.95)$, those aged $80-89$ years had a RR of 0.83 (95\% CI $0.81-0.85$ ), and those aged $\geq 90$ years had a RR of $0.61(95 \%$ CI $0.55-0.68)$. Female patients had a higher chance of treatment success with an aRR of $1.03(95 \%$ CI 1.02-1.04) compared with male patients (Table 1). Crude and aRR models showed a similar trend. Significant differences in mortality between the 4 age-subgroups and sex were found $(P<0.001)$. Compared with patients aged $60-69$ years, those aged 70-79 years had a higher aRR or chance of death at 1.95 (95\% CI 1.78-2.15), those aged 80-89 years had a RR of 3.58 (95\% CI 3.24-3.94), and those aged $\geq 90$ years had a RR of 6.19 (95\% CI 5.26-7.28). Female patients with PTB had a lower mortality than male patients $(\mathrm{aRR}=0.78,95 \% \mathrm{CI}$ 0.72-0.85) (Table 2).

\section{Discussion}

The present study compared patients from 2 cohorts that represented data from 2 sources, the TBCM and NHSO. Data collection in the TBCM program started in 2014, while data collection in the NHSO program started in 2010. The numbers of the PTB cases in the 2 databases are different, 11,869 in NHSO and 8,301 in TBCM. This is because of the transposition of the PTB reporting system of the TBCM that included incremental coverage from hospitals across the country. The completeness of the data sets in the 2 databases seemed to differ; the data completeness of TBCM was $87.8 \%(8,301 / 9,451)$, while that of the NHSO was $72.7 \%(11,869 / 16,335)$. However, if focusing on elderly with new positive acid-fast bacilli sputum smears and excluding patients whose subsequent diagnosis changed to not to be TB, $8,588(=8,633-45)$ in the TBCM cohort and $15,723(=15,833-110)$ in the NHSO cohort, the patients with no treatment outcome reported in the TBCM cohort were $3.3 \%(287 / 8,588)$ and those in the NHSO cohort were $2.5 \%(3,854 / 15,723)$. This might have been because the NHSO has implemented PTB patient monitoring mechanisms, while the TBCM simply accumulated routine data from TB clinics or hospitals. The NHSO has a reimbursement mechanism for local health care entities to conduct routine home visits. Thus, the NHSO could be expected to have more complete information on case management and outcomes. 


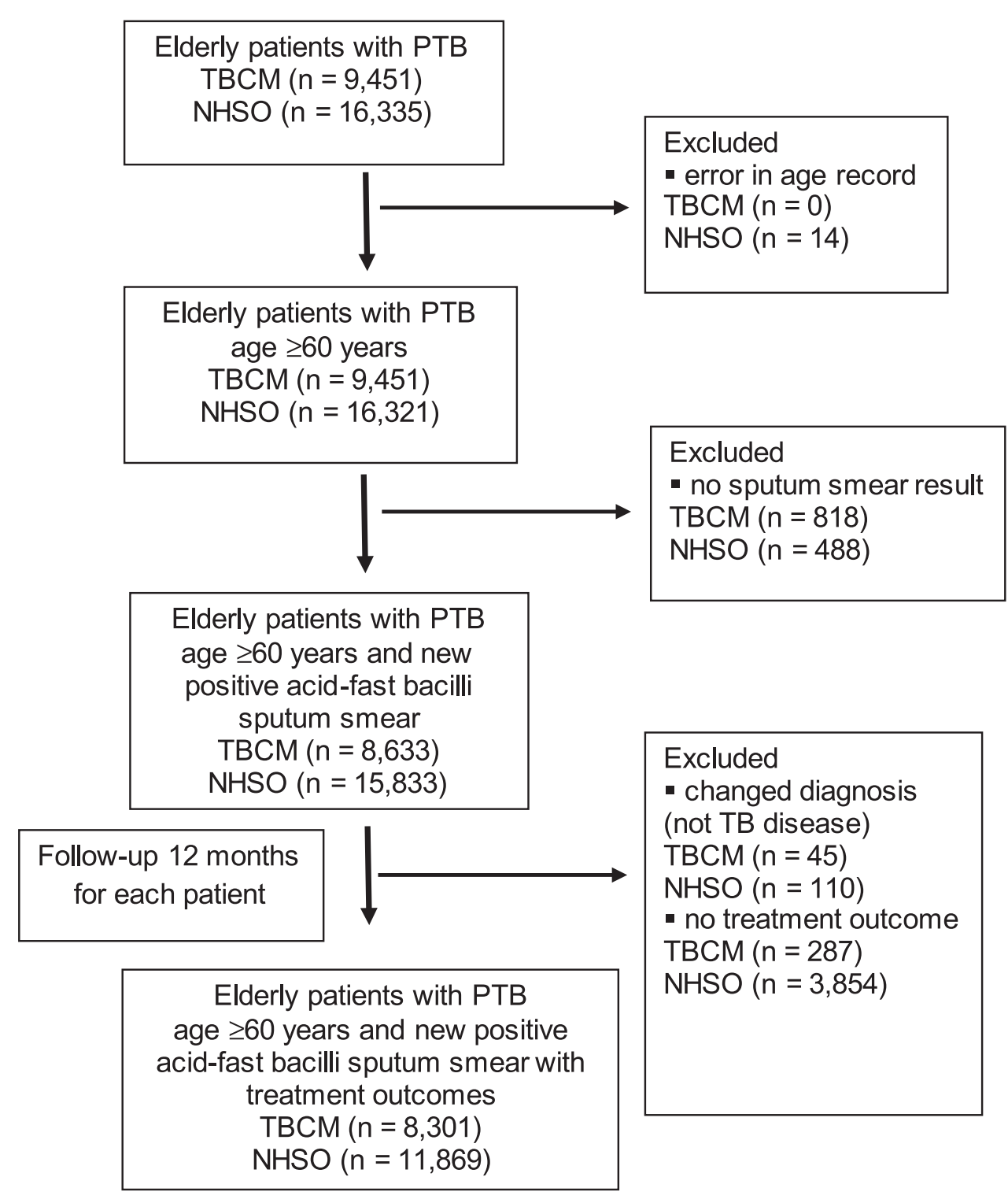

Figure 1. Diagram of elderly patients with pulmonary tuberculosis (PTB) in Thai TB Case Management (TBCM) database from October 1, 2013, to September 30, 2015, and in Thai National Health Security Office (NHSO) database from October 1, 2009, to September 30, 2011

By contrast, the TBCM collected only routine data in the health care database system.

This study showed the overall treatment success rates among all elderly patients with new positive sputum smears indicating TB differed for the 2 periods. The treatment success was $87.5 \%$ among elderly patients with PTB in 2010-2011 (NHSO cohort) against $78.5 \%$ among elderly patients with PTB in 2014-2015 (TBCM cohort). By contrast, the overall mortality among elderly TB patients aged $\geq 60$ years was $8.2 \%$ in the NHSO cohort and $16 \%$ in the TBCM cohort. These findings were unanticipated because the success rates appeared to be lower and mortality appeared to be higher in the cohort covering the more recent time (TBCM) compared with those in the historical cohort (NHSO). The differences may be due to the differences in case management and reporting mechanisms between the TBCM and NHSO as mentioned earlier. The NHSO reimburses payment to hospitals that conduct patient home visits until the end of treatment and complete PTB reports submitted to the NHSO, but the reporting of patients with PTB registered to the TBCM program of the BTB has no reimbursement mechanism. Thus, the more recent statistics on treatment outcomes based on TBCM dataset in 2014-2015 as shown here may reflect a less accurate picture of the TB situation for the elderly in Thailand. As TBCM databases have been in transition to become national databases and NHSO databases have been phased out, we recommend maintaining the treatment outcome monitoring mechanism as previously employed by the NHSO. For Thailand and elsewhere, 


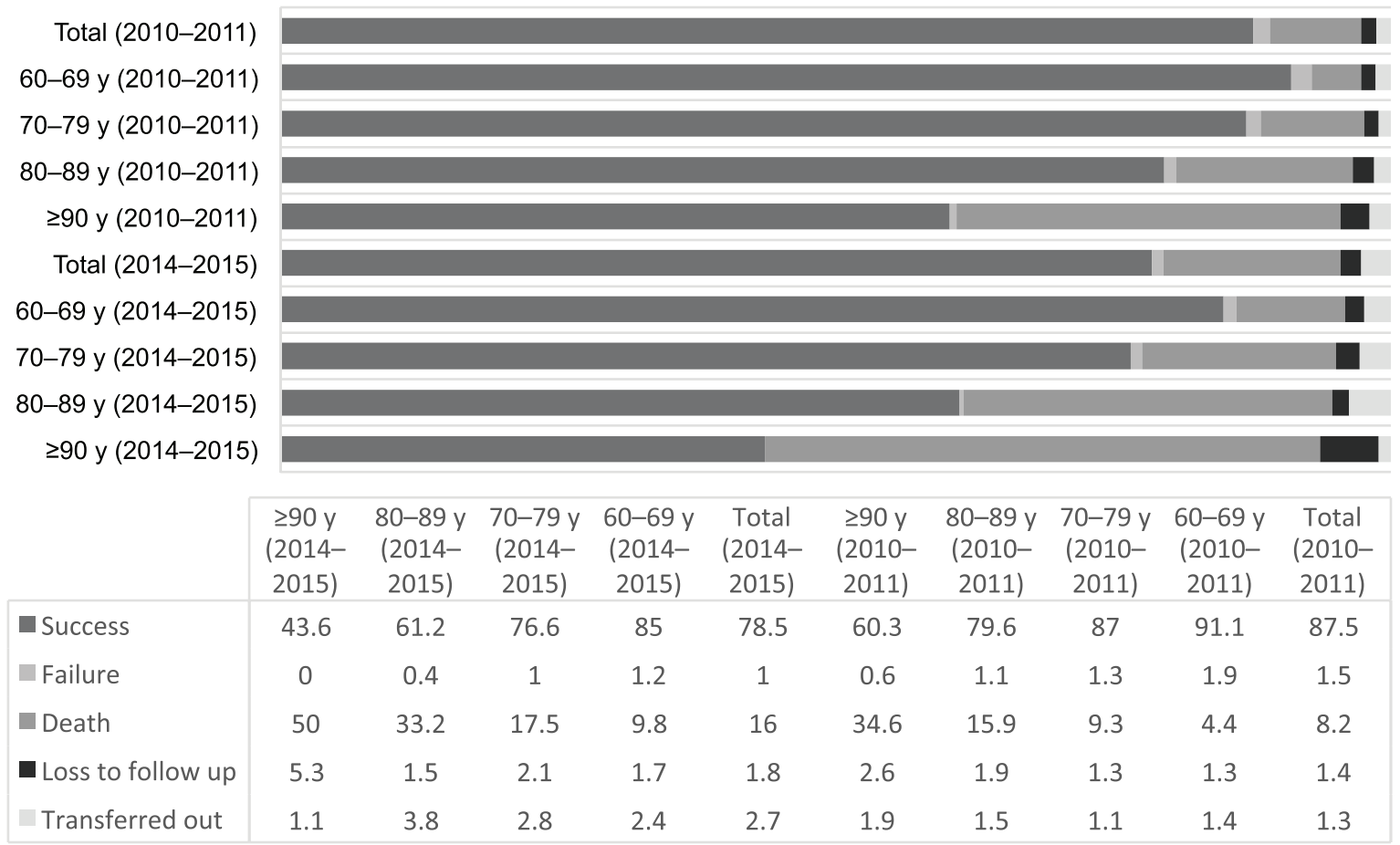

Figure 2. Distribution of treatment outcomes by age group (in years) for elderly patients with pulmonary tuberculosis in 2010-2011 and 2014-2015

Table 1. Factors associated with treatment success among elderly patients with PTB in Thailand, 2010-2011 and 2014-2015

\begin{tabular}{|c|c|c|c|c|c|}
\hline Variable & Total assessed, $\mathbf{N}$ & Success, n (\%) & Nonsuccess, n (\%) & RR, $(95 \% \mathrm{Cl})$ & aRR, $(95 \% \mathrm{Cl})$ \\
\hline \multicolumn{6}{|l|}{ Year } \\
\hline 2010-2011 & 11,869 & $10,388(87.5)$ & $1,481(12.5)$ & 1 & 1 \\
\hline 2014-2015 & 8,301 & $6,519(78.5)$ & $1,782(21.5)$ & $0.90(0.89-0.91)^{*}$ & $0.91(0.90-0.92)^{*}$ \\
\hline \multicolumn{6}{|c|}{ Age group, y } \\
\hline $60-69$ & 10,109 & $8,943(88.5)$ & $1,166(11.5)$ & 1 & 1 \\
\hline 70-79 & 6,898 & $5,719(82.9)$ & $1,179(17.1)$ & $0.94(0.93-0.95)^{*}$ & $0.94(0.93-0.95)^{*}$ \\
\hline $80-89$ & 2,913 & $2,110(72.4)$ & $803(27.6)$ & $0.82(0.80-0.84)^{*}$ & $0.83(0.81-0.85)^{*}$ \\
\hline 90 & 250 & $135(54.0)$ & $115(46.0)$ & $0.61(0.54-0.68)^{*}$ & $0.61(0.55-0.68)^{*}$ \\
\hline \multicolumn{6}{|l|}{ Sex } \\
\hline Male & 13,505 & $11,215(83.0)$ & $2,290(17.0)$ & 1 & 1 \\
\hline Female & 6,636 & $5,673(85.5)$ & $963(14.5)$ & $1.03(1.02-1.04)^{*}$ & $1.03(1.02-1.04)^{*}$ \\
\hline
\end{tabular}

${ }^{*} P<0.001$

aRR, adjusted relative risk; $\mathrm{Cl}$, confidence interval; PTB, pulmonary tuberculosis; $\mathrm{RR}$, relative risk

the lesson learned is that an intensive case follow-up approach to monitor treatment outcomes can improve the quality of data within the TB case-based systems and will result in more accurate estimates of disease burden.

Compared with the treatment success rates for TB patients among all age groups as reported by the national TB program, the findings in this study revealed that elderly patients in the 2010-2011 cohort appeared to have similar success rates (elderly: $87.5 \%$ vs. all ages: $86.0 \%$ (2010) and $85.0 \%$ (2011)), but elderly in the 2014-2015 cohort appeared to have lower success rates (elderly: $78.5 \%$ vs. all ages: $82.3 \%$ (2014) and $80.9 \%(2015)$ ). The mortality for all age groups of TB patients was slightly lower than that of the elderly in the 2010-2011 cohort (elderly: $8.2 \%$ vs. all ages: $7.0 \%$ (2010) and $7.0 \%$ (2011)) but much lower than that of the elderly in the 2014-2015 cohort (elderly: $16.0 \%$ vs. all ages: $7.7 \%$ (2014) and 8.0\% (2015)). When examining the treatment outcomes in details of the cases with complete 
Table 2. Factors associated with death among elderly patients with PTB in Thailand, 2010-2011 and 2014-2015

\begin{tabular}{llllll}
\hline Variable & Total assessed, $\mathbf{N}$ & Death, $\mathbf{n}(\%)$ & Not death, $\mathbf{n}(\%)$ & RR $(\mathbf{0 . 9 5 \%} \mathbf{C l})$ & aRR (0.95\% Cl) \\
\hline $\begin{array}{l}\text { Year } \\
2010-2011\end{array}$ & 11,869 & $976(8.2)$ & $10,893(91.8)$ & 1 & 1 \\
2014-2015 & 8,301 & $1,325(16.0)$ & $6,976(84.0)$ & $1.94(1.80-2.10)^{*}$ & $2.01(1.86-2.17)^{*}$ \\
Age group, $\mathbf{y}$ & & & & & 1 \\
$60-69$ & 10,109 & $677(6.7)$ & $9,432(93.3)$ & 1 & $1.95(1.78-2.15)^{*}$ \\
$70-79$ & 6,898 & $865(12.5)$ & $6,033(87.5)$ & $1.87(1.70-2.06)^{*}$ & $3.58(3.24-3.94)^{*}$ \\
$80-89$ & 2,913 & $658(22.6)$ & $2,255(77.4)$ & $3.37(3.05-3.72)^{*}$ & $6.19(5.26-7.28)^{*}$ \\
$\geq 90$ & 250 & $101(40.4)$ & $149(59.6)$ & $6.03(5.10-7.13)^{*}$ & \\
Sex & & & & & 1 \\
Male & 13,505 & $1,612(11.9)$ & $11,893(88.1)$ & & $0.78(0.72-0.85)^{*}$ \\
Female & 6,636 & $681(10.3)$ & $5,955(89.7)$ & $0.86(0.79-0.94)^{*}$ & 0.78 \\
\hline
\end{tabular}

${ }^{*} P<0.001$

aRR, adjusted relative risk; $\mathrm{Cl}$, confidence interval; PTB, pulmonary tuberculosis; $\mathrm{RR}$, relative risk

data in the 2 cohorts, the loss to follow-up rates were not significantly different: $1.8 \%$ among elderly TB patients in the TBCM cohort and $1.4 \%$ among elderly TB patients in the NHSO cohort. These findings may not be related to the quality of the data in the databases but support good care to increase compliance and successful treatment for elderly patients with PTB.

Nevertheless, from both data sources at 2 time periods, the results suggested that patients with PTB, aged 60-69 years, had a high treatment success rate and low mortality. By contrast, patients with PTB aged $\geq 90$ years had low treatment success rates and high mortality. This finding is consistent with other studies conducted in Asian and Europe where treatment success was associated with younger age [20-22]. Mortality of the elderly was reported as a factor influencing treatment outcomes [23]. Advanced age is obviously a significant risk factor for unsuccessful treatment because advanced age itself contributes to higher mortality, in part through comorbidities [20]. As reported in other studies, mortality showed a substantial increase with age [24]. As Thailand has sought to increase treatment success rates for elderly patients with PTB, those with older age have increasing risks and should receive more attention. Intensive home visits with good care of elderly patients might improve the treatment success rate for PTB [20].

Elderly women with TB had a higher treatment success rate $(85.5 \%)$ and lower mortality $(10.3 \%)$ than elderly men with TB. Similar findings have been observed by others [25]; treatment success for women was 1.4 times higher than that for men [26]. European surveillance data showed that male patients with TB had an approximately 50\% higher risk of death [27]. The higher risk in male patients was explained as the consequence of low compliance with anti-TB therapy, leading to repeated, short interruptions of treatment or a greater loss to follow-up. Improved case-holding strategies may improve treatment outcomes [27-29].

This study employed a retrospective cohort design and was conducted using secondary data compiled from 2 data sources. As with other studies, when using data collected through time, one should be cautious in drawing conclusions in that the results were based on the assumptions regarding the completeness and validity of the data in the databases. As the data from the 2 sources were those compiled from different periods, it would be unfair to make claims as changes overtime.

\section{Conclusions}

The chances of treatment success and risk of mortality were associated with increasing age of elderly TB patients. Higher treatment success rates were found for elderly aged 60-69 years, and higher mortality was found for elderly aged $\geq 90$ years. Patients of advanced age were less likely to achieve treatment success. Female patients had higher antituberculosis treatment success and lower mortality than male patients; women generally had better treatment compliance. We suggest increased awareness of disease progression and better treatment support for those with advanced age. The completeness of the data in the 2 databases seemed to differ; this might have been because the NHSO has implemented PTB patients monitoring mechanisms, while the TBCM simply accumulated routine data. Frequent routine home visits to elderly patients would not only furnish a mechanism to ensure complete treatment and care information for patients with PTB be reported to the national databases but also provide the necessary support and increase the treatment success rate for elderly patients with PTB. 
Author contributions. WS and JK contributed to the conception and design of the study. WS collected and analyzed the data, and all authors contributed to its interpretation. WS drafted the manuscript, and SL, YK, WM, and JK critically revised it. All authors approved the final submitted version and take responsibility for all statements made in the published article.

Acknowledgments. We thank all health officers at the Thai NHSO and the BTB for their generous support. This research did not receive any specific grant from funding agencies in the public, commercial, or not-for-profit sectors.

Conflict of interest statement. The authors have each completed and submitted an International Committee of Medical Journal Editors Form for Disclosure of Potential Conflicts of Interest. None of the authors have any potential conflict of interest to disclose.

\section{References}

[1] World Health Organization. Global tuberculosis control: WHO report 2011. Geneva: WHO Press; 2011.

[2] World Health Organization. Global tuberculosis report 2016. Geneva: WHO Press; 2016.

[3] Bureau of Tuberculosis (National TB Program of Thailand), Ministry of Public Health, Thailand. Data from the TB 07 report [online]. 2007 [cited 2011 Jun 01]; Available from: https://www. tbthailand.org [in Thai]

[4] Bureau of Tuberculosis (National TB Program of Thailand), Ministry of Public Health, Thailand. Data from the TB 07 report [online]. 2007 [cited 2017 May 01]; Available from: https:// tbcmthailand.net/uiform/DashboardTB.aspx [in Thai]

[5] Rizvi N, Shah RH, Inayat N, Hussain N. Differences in clinical presentation of pulmonary tuberculosis in association with age. J Pak Med Assoc. 2003; 53:321-3.

[6] Mackay AD, Cole RB. The problems of tuberculosis in the elderly. Q J Med. 1984; 212:497-510.

[7] Tuberculosis in old age. Tubercle 1983; 64:6971.

[8] Wang C-S, Chen H-C, Yang C-J, Wang W-Y, Chong I-W, Hwang J-J, Huang M-S. The impact of age on the demographic, clinical, radiographic characteristics and treatment outcomes of pulmonary tuberculosis patients in Taiwan. Infection. 2008; 36:335-40.

[9] Leung CC, Yew WW, Chan CK, Chau CH, Tam CM, Lam CW, et al. Tuberculosis in older people: a retrospective and comparative study from Hong Kong. J Am Geriatr Soc. 2002; 50:1219-26.

[10] Lee JH, Han DH, Song JW, Chung HS. Diagnostic and therapeutic problems of pulmonary tuberculosis in elderly patients. J Korean Med Sci. 2005; 20:784-9.

[11] Jeong JI, Jung BH, Kim MH, Lim JM, Ha DC, Cho S-W, Rhui DS. The influence of adverse drug reactions on first-line anti-tuberculosis chemotherapy in the elderly patients. Tuberc Respir Dis. 2009; 67:325-30.
[12] Ai X, Men K, Guo L, Zhang T, Zhao Y, Sun X, et al. Factors associated with low cure rate of tuberculosis in remote poor areas of Shaanxi Province, China: a case control study. BMC Public Health. 2010; 10:112. doi: 10.1186/1471-2458-10-112

[13] Department of Disease Control, Ministry of Public Health. Report of Tuberculosis Program Performance in Thailand, 2009-2015. Bangkok: Bureau of Tuberculosis; 2016. [in Thai]

[14] Bureau of Tuberculosis (National TB Program of Thailand), Ministry of Public Health, Thailand. The data from TB 08 report [online]. 2008 [cited 2018 Mar 01]. Available from: https:// tbcmthailand.net/uiform/DashboardTB.aspx [in Thai]

[15] World Health Organization. Definitions and reporting framework for tuberculosis - 2013 revision (updated December 2014). Geneva: WHO Press; 2014.

[16] World Health organization. Management of tuberculosis: training for health facility staff. H: Monitor TB case detection and treatment. 2nd ed. Geneva: WHO Press; 2010.

[17] World Health Organization. Global tuberculosis control: WHO report 2010. Geneva: WHO Press; 2010

[18] World Health Organization. Treatment of tuberculosis: guidelines. 4th ed. Geneva: WHO Press; 2010.

[19] Cummings P. Methods for estimating adjusted risk ratios. Stata J. 2009; 9:175-96.

[20] Bao Q-S, Du Y-H, Lu C-Y. Treatment outcome of new pulmonary tuberculosis in Guangzhou, China 1993-2002: a register-based cohort study. BMC Public Health. 2007; 7:344. doi: 10.1186/14712458-7-344

[21] Talay F, Kumbetli S, Altin S. Factors associated with treatment success for tuberculosis patients: a single center's experience in Turkey. Jpn J Infect Dis. 2008; 61:25-30.

[22] Farah MG, Tverdal A, Steen TW, Heldal E, Brantsaeter AB, Bjune G. Treatment outcome of new culture positive pulmonary tuberculosis in Norway. BMC Public Health. 2005; 5:14. doi: 10.1186/14712458-5-14.

[23] Tam CM, Leung CC, Noertjojo K, Chan SL, Chan-Yeung M. Tuberculosis in Hong Kong-patient characteristics and treatment outcome. Hong Kong Med J. 2003; 9:83-90.

[24] Research Institute of Tuberculosis; JATA. Tuberculosis annual report 2009. Series 4. Elderly TB. Kekkaku. 2011; 86:737-41. [in Japanese, English abstract]

[25] Okanurak K, Kitayaporn D, Akarasewi P. Factors contributing to treatment success among tuberculosis patients: a prospective cohort study in Bangkok. Int J Tuberc Lung Dis. 2008; 12:1160-5.

[26] Falzon D, Le Strat Y, Belghiti F, Infuso A; EuroTB Correspondents. Exploring the determinants of treatment success for tuberculosis cases in Europe. Int J Tuberc Lung Dis. 2005; 9:1224-9.

[27] Lefebvre N, Falzon D. Risk factors for death among tuberculosis cases: analysis of European surveillance data. Eur Respir J. 2008; 31:1256-60.

[28] Shen X, DeRiemer K, Yuan Z, Shen M, Xia Z, Gui X, et al. Deaths among tuberculosis cases in Shanghai, China: who is at risk? BMC Infectious Diseases. 2009; 9:95. doi: 10.1186/1471-2334-9-95

[29] Balasubramanian R, Garg R, Santha T, Gopi PG, Subramani R, Chandrasekaran V, et al. Gender disparities in tuberculosis: report from a rural DOTS programme in south India. Int J Tuberc Lung Dis. 2004; 8:323-32. 